\title{
Theranostic Nanoemulsions for Macrophage COX-2 Inhibition in a Murine Inflammation Model
}

\section{Authors}

Sravan Kumar Patel ${ }^{\mathrm{a} \ddagger}$, Wissam Beaino ${ }^{\mathrm{b} \ddagger}$, Carolyn J. Anderson ${ }^{\mathrm{b}, \mathrm{c}, \mathrm{d}^{*}}$ and Jelena M. Janjic ${ }^{\mathrm{a}, \mathrm{e}_{*}}$

\section{Affiliations}

${ }^{\mathrm{a}}$ Graduate School of Pharmaceutical Sciences, Mylan School of Pharmacy, Duquesne University, Pittsburgh, PA, 15282, USA

Department of ${ }^{b}$ Radiology, ${ }^{c}$ Pharmacology and Chemical Biology, and ${ }^{\mathrm{d}}$ Bioengineering, University of Pittsburgh, Pittsburgh, PA, 15219, USA

${ }^{\text {e }}$ Chronic Pain Research Consortium, Duquesne University, Pittsburgh, PA, 15218, USA

\section{Corresponding authors}

Jelena M. Janjic, Ph.D.

Graduate School of Pharmaceutical Sciences, Mylan School of Pharmacy, Duquesne University, Pittsburgh, PA, 15282, USA

Fax: 412-396-4660; Email: janjicj@duq.edu

Carolyn J. Anderson, Ph.D.

Department of ${ }^{\mathrm{b}}$ Radiology, ${ }^{\mathrm{c}}$ Pharmacology and Chemical Biology, and ${ }^{\mathrm{d}}$ Bioengineering, University of Pittsburgh, Pittsburgh, PA, 15219, USA

Fax: 412-624-2598; Email: cja34@pitt.edu

* J.M.J. and C.J.A. share senior authorship.

\$ These authors contributed equally to this work 


\begin{abstract}
Targeting macrophages for therapeutic and diagnostic purposes is an attractive approach applicable to multiple diseases. Here, we present a theranostic nanoemulsion platform for simultaneous delivery of an anti-inflammatory drug (celecoxib) to macrophages and monitoring of macrophage migration patterns by optical imaging, as measurement of changes in inflammation. The anti-inflammatory effect of the theranostic nanoemulsions was evaluated in a mouse inflammation model induced with complete Freund's adjuvant (CFA). Nanoemulsions showed greater accumulation in the inflamed vs. control paw, with histology confirming their specific localization in CD68 positive macrophages expressing cyclooxygenase-2 (COX-2) compared to neutrophils. With a single dose administration of the celecoxib-loaded theranostic, we observed a reduction in fluorescence in the paw with time, corresponding to a reduction in macrophage infiltration. Our data strongly suggest that delivery of select agents to infiltrating macrophages can potentially lead to new treatments of inflammatory diseases where macrophage behavior changes are monitored in vivo.
\end{abstract}

Key Words: Inflammation; Macrophage; Cyclooxygenase-2; Fluorescence; Imaging 


\section{Introduction}

Inflammation is involved in the pathogenesis of many common diseases [1]. Although it is beneficial in defending the host from injury or infection, unresolved inflammation can have detrimental effects. Methods to effectively intercept pathways leading to chronic inflammation are under intense development. Several cell types such as neutrophils, dendritic cells, and macrophages infiltrate the site of injury. Here we present a novel nanomedicine strategy for direct targeting of infiltrating inflammatory cells (macrophages) for therapeutic and diagnostic purposes.

Macrophages are one of the most abundant cell types present in inflammation, and there is increasing evidence that these cells significantly contribute to the pathogenesis of diseases such as cancer [2], diabetes [3], atherosclerosis [4], and rheumatoid arthritis [5]. Following the shortlived neutrophils, macrophages populate the inflammation sites where they participate in the removal of pathogens, cell debris and senescent cells, modulation of adaptive immunity, tissue remodeling and restoration of homeostasis [6]. If the inflammation is not resolved, the inflammatory mediators produced by macrophages such as prostaglandins, reactive oxygen and nitrogen species, and proteases can cause substantial tissue injury.

Since macrophage infiltration is positively correlated with the severity of the disease [7], macrophages have emerged as prime targets for imaging and treatment in inflammatory-based diseases due to their abundance and involvement in disease development. The ability to image macrophages has led to their utilization in disease diagnosis [8], prediction of disease severity [9] and evaluation of the therapeutic efficacy [10]. 
Nanoparticles have been utilized to deliver imaging and therapeutic agents to macrophages with or without targeting ligands, as they are readily internalized due to macrophages' natural phagocytic ability ("big eaters"). Nanoparticles that have imaging and therapeutic agents incorporated into their structure allow concurrent therapy and diagnosis, and thus are often referred to as "theranostics". Several macrophage-targeted theranostics have been reported in the literature (for a recent review, see [11]). Light-activated theranostic nanoparticles have been investigated for selective ablation of macrophages using photodynamic and photothermal therapies [12]. Similarly, theranostics incorporating chemotherapeutic drugs were utilized for depletion of macrophages, reduction of pro-inflammatory mediators [13], or alteration of their phenotype [14]. In the majority of the studies, imaging was utilized either for visualization of diseased tissue such as tumors, arthritic joints, atherosclerotic plaques, or in the case of photobased applications, to guide therapy [12]. Theranostic nanoparticles have also been employed to visualize the therapeutic response after subsequent administration of the theranostic [15]. To our knowledge, visualizing the pharmacological response after a single administration of a theranostic nanoparticle to macrophages has not been reported.

Our goal was to design, develop and evaluate fluorescent perfluorocarbon (PFC) theranostic nanoemulsions for macrophage imaging and monitoring of therapeutic efficacy after administration of a single dose. PFC nanoemulsions have been applied in in situ labeling of macrophages and visualizing their accumulation in diseases such as arthritis [10], inflammatory bowel disease [16], cancer [17], neuroinflammation [18], and pulmonary inflammation [19]. Our lab and others have also reported dual mode nanoemulsions that incorporate fluorescent dyes and PFCs for macrophage imaging $[17,18,20,21]$ and also on the in vitro utility of nanoemulsions with therapeutic capabilities [21]. Here we report the development and in vivo application of an 
anti-inflammatory drug-loaded fluorescent PFC nanoemulsion (to be referred to as "theranostic nanoemulsion"). The theranostic nanoemulsion incorporates a cyclooxygenase-2 (COX-2) inhibitor, celecoxib. Activated macrophages in inflammation are one of the key cells expressing COX-2, an inducible pro-inflammatory enzyme involved in the pathogenesis of several diseases [22-24]. COX-2 is involved in the synthesis of prostaglandin E2 (PGE2), a potent inflammatory mediator responsible for pain, increased vascularity, blood flow and recruitment of leukocytes to the inflamed site [25-27]. In this report, we demonstrate that by employing fluorescently labeled nanoemulsions loaded with celecoxib, macrophage infiltration and inflammation can be reduced, and this process can be monitored by optical imaging in vivo. As such, nanoemulsions taken up into macrophages can effectively change their behavior, leading to therapeutic effects by inhibiting production of key pro-inflammatory mediators in these cells. The macrophages are therefore potentially converted into immuno-therapeutic cells, as their function that perpetuates inflammatory processes is reduced. One can envision that additional inflammatory drugs can be delivered using theranostic nanoemulsions, and select pathways inside these cells can be inhibited or modulated for therapeutic effects. Therefore, the work described here sets the stage for a novel type of inflammation treatment, with macrophages being the therapeutic and diagnostic targets.

\section{Materials and Methods}

\subsection{Materials}

All chemicals and cell culture reagents were obtained from commercial sources and used without purification. Celecoxib was purchased from LC Laboratories, Woburn, MA, USA. Perfluoropolyethylene glycol ether (PFPE) was obtained as a generous gift from Celsense Inc., 
Pittsburgh, PA, USA. Pluronic ${ }^{\circledR}$ P105 was obtained from BASF, Cremophor EL ${ }^{\circledR}(\mathrm{CrEL})$ was obtained from Sigma Aldrich. 1,1'-Dioctadecyl-3,3,3',3'-Tetramethylindodicarbocyanine (DiD) dye was purchased from Life Technologies. Bacterial lipopolysaccharide (LPS) and cytochalasin B were obtained from Sigma Aldrich. The CellTiter-Glo® cell viability assay kit and Griess Reagent System for nitrite measurements were purchased from Promega, Madison, WI, USA. Phycoerythrin conjugated Rat anti-mouse CD86 (CD86-PE) antibody and Rat IgG $_{2 \mathrm{a}, \mathrm{k}}$ conjugated to PE (isotype control) were obtained from BD Biosciences, San Jose, CA. The murine macrophage cell line RAW 264.7 (TIB-71) was obtained from ATCC. Cells were culture as previously reported [21]. Unless specified, cells were incubated at $37{ }^{\circ} \mathrm{C}$ in a humidified atmosphere containing $5 \% \mathrm{CO}_{2}$.

\subsection{Preparation and characterization of fluorescent PFPE nanoemulsions}

\subsubsection{Preparation of nanoemulsions}

Nanoemulsion formulation components and their amounts are shown in Table 1. Nanoemulsions were prepared as reported earlier [21] with some modifications. Celecoxib (5 mg) was solubilized in neat hydrocarbon oil, Miglyol $810 \mathrm{~N}$ (0.95 g), by overnight stirring. For drug-free nanoemulsions, neat Miglyol 810N with or without the fluorescent dye was used. For dye-loaded nanoemulsions, DiD dye (100 $\mu \mathrm{L}$ in $2.5 \mathrm{mM}$ ethanol stock) was added to Miglyol $810 \mathrm{~N}$ with or without the solubilized drug and vortexed in the dark. PFPE, in required amounts (Table 1), was added and vortexed followed by the addition of surfactant mixture in $2 \mathrm{~mL}$ portions. The surfactant mixture contained 3\% w/v CrEL and 2\% w/v Pluronic ${ }^{\circledR}$ P105 dissolved in de-ionized water. After the addition of deionized water, the mixture was vortexed and processed on a microfluidizer (Microfluidics, M110S) for 30, 38 or 46 pulses at 6 bar inlet pressure. The total 
volume of the prepared nanoemulsions was $25 \mathrm{~mL}$. The obtained nanoemulsion was sterile filtered $\left(0.22 \mu \mathrm{m}\right.$, Millipore) after equilibration for one day at $4{ }^{\circ} \mathrm{C}$. $\mathrm{pH}$ of the drug-free nanoemulsion (DFNE) and celecoxib-loaded nanoemulsion (CXBNE) were recorded after filtration.

\subsubsection{Colloidal characterization}

Dynamic light scattering (Zetasizer Nano ZS, Malvern Instruments, UK) was used to measure droplet size at $25^{\circ} \mathrm{C}$. Nanoemulsion was dispersed in de-ionized water at 1:40 dilution. Zeta potential was recorded on the same sample in standard zeta potential cells fitted with electrodes. Every sample was analyzed three times and each measurement was run for at least 12 times. To assess serum stability, nanoemulsions were dispersed in complete cell culture media at 1:40 dilution, stored at $37{ }^{\circ} \mathrm{C}$, and the sizes were recorded without further dilution. Similarly, nanoemulsions were dispersed in buffered solutions at $\mathrm{pH} 5.0$ and 7.4 at 1:40 dilution, incubated at $37{ }^{\circ} \mathrm{C}$ and sizes recorded at predetermined intervals. Samples for follow-up stability analysis were stored at 4, 25 and $37{ }^{\circ} \mathrm{C}$. For nanoemulsion formulation optimization studies, samples were also stored at $60{ }^{\circ} \mathrm{C}$ for one month and analyzed for droplet size and polydispersity.

\subsubsection{Drug loading}

Drug loading in the nanoemulsions was assessed using a previously validated high-performance liquid chromatography (HPLC) method [21]. Briefly, $50 \mu \mathrm{L}$ of nanoemulsion was dispersed in methanol in a glass centrifuge tube and vortexed for $1 \mathrm{~min}$. This dispersion was subjected to centrifugation at $2000 \mathrm{rpm}$ for $5 \mathrm{~min}$ at $4{ }^{\circ} \mathrm{C}$ to separate PFPE oxide. The supernatant was assayed without further dilution in triplicate using HPLC. The absorption maximum of celecoxib in nanoemulsion was $252 \mathrm{~nm}$ with retention time at $3.8 \mathrm{~min}$ (Fig. S1). 


\subsubsection{Nanoemulsion characterization for fluorescent properties}

To assess fluorescent properties, nanoemulsions were diluted with de-ionized water $(8 \% \mathrm{v} / \mathrm{v})$ and $150 \mu \mathrm{L}$ of this dispersion was measured for fluorescence excitation and emission on Tecan Saffire $^{2}$ plate reader using a $10 \mathrm{~nm}$ bandwidth. Emission spectrum was obtained by measuring fluorescence intensity from $630-798 \mathrm{~nm}$ with $4 \mathrm{~nm}$ step size using excitation wavelength of 610 $\mathrm{nm}$. The excitation spectrum was obtained by measuring fluorescence intensity for wavelengths 400-668 nm with $4 \mathrm{~nm}$ step size using emission wavelength of $690 \mathrm{~nm}$.

\subsection{In vitro cell culture studies}

\subsubsection{In vitro cytotoxicity}

The effect of nanoemulsions on the viability of RAW 264.7 macrophages was evaluated after a $24 \mathrm{~h}$ incubation at $37{ }^{\circ} \mathrm{C}$ with different concentrations of nanoemulsion dispersed in whole media. Control cells were not exposed to any treatments. Cells were plated at 10,000/well in 96 well plates and incubated overnight for adhesion, followed by $24 \mathrm{~h}$ incubation with treatments. After incubation, cells were washed twice with DPBS (1x). CellTiter-Glo® analyte was added (40 $\mu \mathrm{L} /$ well) to induce cell lysis by shaking in the dark for 20 min at RT. The obtained cell lysates $(80 \mu \mathrm{L})$ were transferred to a white opaque plate and luminescence recorded on microplate reader Victor 2 (Perkin Elmer, Waltham, MA).

\subsubsection{CD86 expression and nitric oxide release}

In order to evaluate if nanoemulsion delivery affect any inflammatory mediators, nitric oxide release and CD86 cell surface markers were assessed in macrophages. Cells were incubated with DFNE or CXBNE (9.3 $\mu$ M celecoxib) overnight. Cells exposed to LPS (200 $\mathrm{ng} / \mathrm{mL})$ were used 
as positive control, while the negative control cells were left untreated. Supernatants were analyzed for nitric oxide concentration using commercially available Griess reagent according to manufacturer instructions. Cells were collected by trypsinization and labeled with PE-conjugated anti-CD86 antibody or isotype control. After repeated washings with centrifugation (300g, 5 min) in PBS / 2\% FBS, cells were fixed with 2\% paraformaldehyde, washed and resuspended in PBS. Samples were analyzed using flow cytometry (BD TMAccuri) and 50,000 events were recorded. Nanoemulsion was detected in FL4, while CD86 fluorescence was recorded in FL2 channel. Gating was applied based on forward scatter (FSC) and side scatter (SSC) as shown in Fig. S2. The mean fluorescence of gated cells was utilized for analysis.

\subsubsection{Cellular uptake}

Time and concentration dependent uptake kinetics of nanoemulsions were determined in macrophages. RAW 264.7 cells were grown in 6-well plates $\left(0.3 \times 10^{6} /\right.$ well), incubated overnight for attachment, and followed after exposure to different doses of nanoemulsion $(0,2$, 4, $6,8 \mathrm{mg} / \mathrm{mL}$ PFPE) for $24 \mathrm{~h}$ in triplicates. Cells were trypsinized and washed by centrifugation. Cells were lysed using lysing buffer containing Triton-X100 (1\%) (Sigma-Aldrich). A portion of the lysate was used for measuring protein content using Bradford protein assay (Bio-Rad, Hercules, CA). Cell lysates $(150 \mu \mathrm{L})$ were transferred to a clear bottom 96-well plate and fluorescence intensity was recorded on a plate reader (Saffire 2, Tecan) at excitation wavelength of $610 \mathrm{~nm}$ and emission wavelength of $670 \mathrm{~nm}$. The obtained fluorescence intensity was

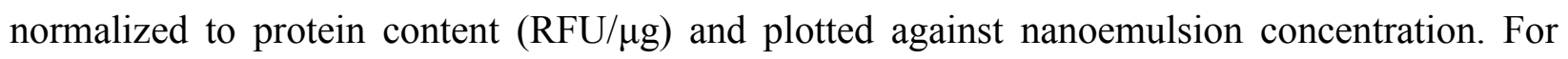
time-dependent uptake, cells were grown in 12-well plates $\left(0.25 \times 10^{6} /\right.$ well). After overnight incubation, cells were exposed to nanoemulsion at single dose $(4.2 \mathrm{mg} / \mathrm{mL})$ for different time points $(5,15,30,60,120,240,360$ and $420 \mathrm{~min})$ in triplicate. Cells were collected by 
trypsinization, resuspended in PBS / 2\% FBS, and washed twice by centrifugation (300g, $5 \mathrm{~min}$ ). Cells were fixed with 2\% PFA and analyzed by flow cytometry (BD AccuriтM). 20,000 events were recorded in the gated region (Fig. S2) and the nanoemulsion was detected in FL4. Data were plotted as mean fluorescence at each time point for triplicate samples.

To assess the effect of phagocytosis inhibition on uptake, cells were plated in 12 well plates $(0.2$ x $10^{6}$ cells) and left for attachment overnight. After aspiration of media and washing with DPBS, cells were exposed to different doses of nanoemulsion with or without cytochalasin B ( $5 \mu \mathrm{g} / \mathrm{mL})$ for $2.5 \mathrm{~h}$ in triplicates. Cells were collected by trypsinization, washed twice by centrifugation (300g, $5 \mathrm{~min}, 4^{\circ} \mathrm{C}$ ) in DPBS / 2\% FBS, and fixed with 2\%PFA. Cells resuspended in DPBS were analyzed by flow cytometry (BD Accuri ${ }^{\mathrm{TM}}$ ). Nanoemulsion was detected in FL4 and 30,000 events per sample were collected in the gated region (Fig. S2).

\subsubsection{Confocal microscopy}

RAW 264.7 macrophages $\left(0.2 \times 10^{6}\right.$ cells $)$ were seeded on glass coverslips in a 24 well plate and left for adhesion overnight. Cells were then incubated with nanoemulsion $(30 \mu \mathrm{L})$ in culture media $(1 \mathrm{~mL})$ for two hours at $37{ }^{\circ} \mathrm{C}$. Cell were then washed with DPBS and fixed with $4 \%$ paraformaldehyde (PFA) for 20 min at room temperature (RT) and stained with DAPI for nuclei visualization. For kinetic studies, cells were incubated with nanoemulsion $(30 \mu \mathrm{L}$ in $1 \mathrm{~mL}$ medium) for $5,15,30$ and 60 min at 4 and $37^{\circ} \mathrm{C}$, fixed with $4 \%$ PFA, and stained with DAPI. After washing with DPBS, coverslips were mounted using Diamond anti-fading medium (Invitrogen, Grand Island, NY). Fluorescence was monitored using a Zeiss Apotome system equipped with a Zeiss HPO PL APO 63x oil immersion lens (numerical aperture 1.4-0.6). For colocalization with acidic compartments, cells were seeded on a glass bottom dishes and left for 
adhesion. Cells were then incubated with nanoemulsion ( $30 \mu \mathrm{L}$ in $1 \mathrm{~mL}$ medium) for $45 \mathrm{~min}$ at $37^{\circ} \mathrm{C}$, and Lysotracker Green DND-26 $(1 \mu \mathrm{M})$ (Invitrogen, Grand Island, NY) was added for 15 min at $37^{\circ} \mathrm{C}$. Cells were washed with DPBS and immediately imaged using a Zeiss Apotome system equipped with a Zeiss HPO PL APO 63x oil immersion lens (numerical aperture 1.4-0.6).

\subsection{Mouse inflammation model}

All animal studies were performed under the Guide for the Care and Use of Laboratory Animals under the auspices of Division of Laboratory Animal Resources (DLAR) of the University of Pittsburgh. Six to eight week old female SKH1 hairless (immuno-competent) mice were purchased from Charles River Laboratories (Horsham, Pennsylvania) and were injected subcutaneously in the right paw with complete Freund's adjuvant (CFA) (50 $\mu \mathrm{L})$ [28].

\subsection{In vivo studies in CFA-induced paw inflammation mouse model}

SKH1 hairless mice were injected via tail vein with CXBNE $(150 \mu \mathrm{L}, 437.6 \mu \mathrm{M})(\mathrm{n}=5)$ or DFNE $(150 \mu \mathrm{L})$ and free celecoxib dissolved in a surfactant mixture containing CrEL and Pluronic ${ }^{\circledR}$ P105 $(115 \mu \mathrm{L})(\mathrm{n}=5) 12 \mathrm{~h}$ prior to paw inflammation induction induced by CFA. Infiltration of macrophages loaded with nanoemulsion at the inflammation site was monitored by in vivo fluorescence imaging using an IVIS ${ }^{\circledR}$ Lumina XR (Perkin Elmer; Waltham, MA) imaging station using $640 \mathrm{~nm}$ and $700 \mathrm{~nm}$ filters for excitation and emission, respectively. Images were acquired at $2,6,10,24,34,48$ and $72 \mathrm{~h}$ post $\mathrm{CFA}$ injection. Imaging parameters (exposure $1 \mathrm{sec}$, binning medium, lamp high, F/stop 2) were maintained for all time points. During image acquisition, tail and abdominal areas of the mice were covered with a black cloth to reduce background saturation of signal. Images were quantified by drawing a region of interest (ROI) around the inflamed leg and the contralateral control leg (Fig. S3). Total 
fluorescence photon number was calculated after background subtraction. Data are presented as the fluorescence ratio of inflamed to control leg. Mice were sacrificed $(\mathrm{n}=3)$ after the $72 \mathrm{~h}$ time point imaging, and organs were collected and imaged using the IVIS ${ }^{\circledR}$ Lumina XR (Perkin Elmer, Waltham, MA) imaging station, and then weighed. ROIs were drawn around each organ, and total fluorescence photon was calculated and normalized to the organ weight. The total dose was calculated by quantifying the fluorescence of a standard dose imaged with the same imaging parameters.

\subsection{Ex vivo histological analysis}

Following the in vivo studies at $72 \mathrm{~h}$ post-inflammation, mice injected with either CXBNE or DFNE and free celecoxib dissolved in the surfactant mixture were sacrificed and the inflamed paw was collected and snap frozen. In a separate experiment, SKH1 hairless mice were injected with DFNE $(150 \mu \mathrm{L})$, and paw inflammation was induced by injection of CFA $(50 \mu \mathrm{L})$ subcutaneously at the right paw. After $24 \mathrm{~h}$, mice were sacrificed; spleens and paws were collected and snap frozen. Tissues were then embedded in optimal cutting temperature (OCT) medium. $10 \mu \mathrm{m}$ sections were obtained using a cryotome. Section were fixed with $4 \%$ PFA in DPBS for 10 min at RT. Non-specific binding was saturated with $5 \%$ goat serum for $1 \mathrm{~h}$ at RT. Rat anti-Mouse Gr1-FITC (BD bioscience, San Jose, CA) or Rat anti-Mouse CD68 (AbD Serotec, Raleigh, NC) (1/100 dilution in DPBS/0.1\%BSA/0.05\% Tween-20) and Goat antiMouse COX-2 antibodies (abcam ${ }^{\circledR}$, Cambridge, MA) (1/100 dilution in PBS/0.1\%BSA/0.05\% Tween-20) were added overnight at $4{ }^{\circ} \mathrm{C}$ followed by a secondary antibody, Anti-Rat-Alexa 488 and Anti-goat-Alexa 546 (Invitrogen, Grand Island, NY) (1/300 dilution in PBS/0.1\%BSA/0.05\% Tween-20) for $1 \mathrm{~h}$ at RT. After washing in DPBS/0.05\% Tween-20, coverslips were mounted using Diamond anti-fading medium (Invitrogen, Grand Island, NY). 
Immunofluorescence staining was monitored using a Zeiss Apotome system equipped with a Zeiss HPO PL APO 40x or 63x oil immersion lens (numerical aperture 1.4-0.6).

\subsection{Statistical Analysis}

A Two-way repeated-measures ANOVA with Bonferroni post-hoc test was utilized to compare mean fluorescence intensity of groups treated with DFNE or CXBNE at each imaging time point. Imaging time point and treatment were chosen as factors for this analysis. A student's t-test was used to compare mean fluorescence intensity of organs between groups treated with DFNE or CXBNE. Statistical significance level was set at $\mathrm{p}<0.05$ and data was analyzed using GraphPad Prism 6.

\section{Results \& Discussion}

\subsection{Preparation \& characterization of nanoemulsions}

We previously reported PFPE nanoemulsions with dual imaging capabilities $\left({ }^{19} \mathrm{~F}\right.$ MRI and NIRF) [21, 29]. In an attempt to increase imaging efficiency, here we prepared nanoemulsions with greater amount of PFPE. As increasing PFPE content could require a change in surfactant amount and processing conditions for nanoemulsion stability, a Box-Behnken design of experiments was initially proposed which varied the amount of surfactant and PFPE, and the number of pulses in the microfluidizer. However, the full design was not completed as one of the three tested nanoemulsions met the desired criteria (Table 1 and Fig. 1). All nanoemulsions were devoid of size populations showing a narrow size distribution and displayed a low polydispersity index (PDI) of less than 0.15 (Table 1). Nanoemulsions prepared with $2 \% \mathrm{w} / \mathrm{v}$ surfactant (NE2 and NE3) showed lower droplet size compared to 3.75\% w/v (NE1) (Table 1). We observed an increase in average size of approximately $20 \mathrm{~nm}$ in nanoemulsion with increased surfactant 
amount (NE1), while NE2 and NE3 showed comparable average droplet size (Table 1, Fig. 1A). Under degradation tests using elevated temperature $\left(60{ }^{\circ} \mathrm{C}\right)$, size was monitored for at least 2 months in order to select the nanoemulsion with superior stability. NE1 did not show significant increase in size for up to 2 months compared to NE2 and NE3, which increased size within two weeks of high temperature exposure (Fig. 1B), demonstrating high colloidal stability of NE1. To compare storage stability of the nanoemulsions, droplet size was monitored for samples stored at 4, 25, and $37^{\circ} \mathrm{C}$ (Fig. 1C-E). The droplet size for all nanoemulsions tested showed no significant changes at 4 and $25^{\circ} \mathrm{C}$. However, samples stored at $37^{\circ} \mathrm{C}$ showed an increase in size for NE2 and NE3, while NE1 remained stable throughout the test period of one month (Figure 1C-E). All the nanoemulsions showed a negative zeta potential (Table 1). Based on these results, it appears that low oil-to-surfactant $(\mathrm{O} / \mathrm{S})$ ratio is required for high colloidal stability under storage and at elevated temperature. The effect on cell viability was comparable for nanoemulsions with low and high surfactant amounts (Fig. S4). Based on these results, the composition and processing conditions of NE1 were selected as optimized to prepare fluorescent nanoemulsions for in vitro and in vivo studies.

\section{Table 1}

DLS characterization of nanoemulsions NE1, NE2 and NE3

\begin{tabular}{cccccccc}
\hline Code & $\begin{array}{c}\text { PFPE } \\
(\mathbf{\%} / \mathbf{v})\end{array}$ & $\begin{array}{c}\text { Surfactant } \\
(\mathbf{\%} / \mathbf{v})\end{array}$ & Pulses & $\mathbf{O} \mathbf{S}^{\mathbf{a}}$ & $\begin{array}{c}\text { Size } \\
(\mathbf{n m})\end{array}$ & PDI & $\begin{array}{c}\text { Zeta potential } \\
(\mathbf{m V})\end{array}$ \\
\hline NE1 & 18.75 & 3.75 & 38 & 5.96 & 156 & 0.12 & -14.0 \\
NE2 & 18.75 & 2 & 30 & 11.17 & 136 & 0.10 & -12.1 \\
NE3 & 18.75 & 2 & 46 & 11.17 & 133 & 0.10 & -12.8 \\
\hline
\end{tabular}

$* \mathrm{O} / \mathrm{S}=$ Oil to surfactant ratio. Oil phase includes Miglyol $810 \mathrm{~N}$ and PFPE oxide 
The optimized nanoemulsion was modified for fluorescence imaging using a lipophilic fluorescent dye, DiD. The addition of the dye reduced the droplet size by $20-25 \mathrm{~nm}$, probably due to its adsorption in the surfactant layer. A small reduction in zeta potential was also observed compared to dye-free nanoemulsions $(-6 \mathrm{mV}$ vs. $-14 \mathrm{mV})$. There was no influence of drug incorporation on average droplet size and zeta potential (Fig. 2A). The stability of the nanoemulsions was investigated by monitoring average size changes with time at $4{ }^{\circ} \mathrm{C}$ storage, serum exposure, and variation in dispersant $\mathrm{pH}$. Both DFNE and CXBNE displayed high stability under storage at $4{ }^{\circ} \mathrm{C}$ (Fig. 2B). Even after 100 days, the size distribution by intensity showed a single peak demonstrating monomodal distribution (Fig. S5). A small increase in size of less than $15 \mathrm{~nm}$ was noted after 100 days and PDI remained below 0.15 . Incubation of nanoemulsions with serum-containing media showed a small reduction in size (Fig. 2C). However, the serum did not affect the size distribution (Fig. S6). This reduction in size was previously reported for colloidal carriers and it was speculated that the change in dispersant viscosity and refractive index could potentially affect the size analysis with DLS [30]. Average droplet size and size distribution of nanoemulsions remained unaltered after incubation at $\mathrm{pH} 5.0$ and 7.4 for 5 days (Fig. 2D). The $\mathrm{pH}$ of the undiluted nanoemulsions was between 6.75 and 7.1, which is close to physiological $\mathrm{pH}$ of 7.4. These results clearly indicate that the nanoemulsion is stable under different stress conditions and was acceptable for in vitro and in vivo biomedical applications.

Nanoemulsions were characterized by ${ }^{19} \mathrm{~F}$ NMR spectroscopy and fluorescence. ${ }^{19} \mathrm{~F}$ NMR of the nanoemulsions (Fig. S7) showed characteristic peaks similar to the spectrum of pure PFPE oxide [31]. In order to determine the appropriate fluorescence conditions for in vitro and in vivo studies, emission and excitation spectra for DFNE and CXBNE were determined. Excitation and 
emission maximum of $648 \mathrm{~nm}$ and $668 \mathrm{~nm}$, respectively, were observed for both nanoemulsions (Fig. 2E-F). The presence of drug did not show any shift in the emission and excitation spectra. A slight blue shift was observed compared to the free dye (Fig. S8) dissolved in ethanol/water mixture $(654 / 678 \mathrm{~nm})$, possibly due to the presence of surfactants in the nanoemulsion. However, the fluorescence properties remained in the NIR region, which is ideal for in vivo imaging due to the reduced auto-fluorescence.

\subsection{In vitro evaluation in $R A W 264.7$ macrophages}

The optimized nanoemulsion, with and without celecoxib, was tested at different doses in macrophages and did not affect their viability after $24 \mathrm{~h}$ exposure (Fig. 3A). In order to ascertain that these nanoemulsions do not produce any pro-inflammatory effects, nitric oxide (NO) release and CD86 expression changes were monitored after $24 \mathrm{~h}$ of exposure. NO and CD86 were chosen as the markers for macrophage activation. Lipopolysaccharide (LPS) was used as a positive control to induce the pro-inflammatory phenotype in macrophages, which leads to increased CD86 expression and NO release. CD86 expression and NO release were not altered by the nanoemulsions (Fig. 3B and C). CD86 and NO levels were comparable to untreated cells, and significantly lower than LPS-activated cells, which clearly indicate that nanoemulsions do not stimulate pro-inflammatory phenotype in macrophages.

To understand the uptake and intracellular fate of nanoemulsions, cellular uptake studies were conducted in RAW 264.7 macrophages. Confocal microscopy showed that the nanoemulsions were highly internalized by macrophages (Fig. 4B) and localized to acidic endosomal/lysosomal

compartments (Fig. 4B, C). ${ }^{19} \mathrm{~F}$ NMR of cells exposed to nanoemulsion also confirmed the uptake based on characteristic peaks of PFPE (Fig. S9). Two possible mechanisms of 
nanoemulsion internalization exist in macrophages. Nanoemulsions can enter cells either passively through membrane penetration or actively via phagocytosis, endocytosis and pinocytosis [32]. In order to identify the mechanism of internalization, RAW 264.7 macrophages were incubated with the nanoemulsion for 5, 15, 30, and 60 min. At $37^{\circ} \mathrm{C}$, nanoemulsion could be easily detected at the cell membrane as early as $5 \mathrm{~min}$, and the internalization increased over time with a punctuated fluorescent staining pattern indicative of localization in discrete vesicular compartments. At $4{ }^{\circ} \mathrm{C}$, barely detectable levels of nanoemulsion were taken up after 5 min, and we observed minimal internalization after $60 \mathrm{~min}$ (Fig 5A). These results demonstrate predominantly energy-dependent active mechanisms of internalization for the nanoemulsion in macrophages. In addition, dose- and time-dependent uptake of nanoemulsion was observed in macrophages (Fig. 5B-C). The cell-associated fluorescence reached saturation at higher nanoemulsion concentrations, which is typical for an endocytotic pathway of uptake [33]. A linear increase in uptake with time was observed; within $2 \mathrm{~h}$ of exposure, more than $90 \%$ of cells were shown to be positive for nanoemulsion.

One of the primary mechanisms of nanoparticle uptake in macrophages is phagocytosis. To assess if phagocytosis is involved in the internalization of nanoemulsions, cellular uptake was compared in the presence and absence of the phagocytosis inhibitor cytochalasin B. Cellular uptake was significantly lower with cytochalasin B (Fig. 5D). In the presence of cytochalasin B, nanoemulsion uptake was reduced by $70 \%$, and this result was observed at all tested nanoemulsion concentrations, confirming that the uptake is predominantly mediated through phagocytosis.

The pharmacological effect of celecoxib delivery was evaluated by quantification of PGE2 in activated macrophages. Similar to our previously reported results [21], CXBNE produced 
significant reduction in $\mathrm{PGE}_{2}$ produced by LPS-activated macrophages compared to DFNE (Fig. S10). Together, these data strongly suggest that the nanoemulsion, efficiently internalized by macrophages in a dose- and time-dependent manner and inhibiting COX-2 enzyme activity, can produce anti-inflammatory effects in vivo. A paw inflammation mouse model was used to test the in vivo efficacy of these nanoemulsions as imaging and therapeutic agents.

\subsection{In vivo studies in a murine paw inflammation model}

To test the in vivo diagnostic and therapeutic potential of the theranostic nanoemulsion, a murine inflammation model (CFA injection in the sub-plantar region of the paw) was utilized. CFA is an emulsion containing inactivated mycobacterium tuberculosis and it has been shown to induce local inflammation leading to increased leukocyte recruitment [28].

First we evaluated the potential of the nanoemulsion to be delivered to macrophages. Immunofluorescence staining of the inflamed paw and spleen from mice injected with the nanoemulsion shows a strong and major colocalization of the nanoemulsion with macrophages while having undetectable colocalization with neutrophils (Fig. 6). These data show that the nanoemulsion is specifically internalized by macrophages in vivo at the site of inflammation and in spleen and is suitable to image and deliver therapeutics to macrophages. Nevertheless, we cannot exclude the possibility that the nanoemulsion could be also taken up by other antigen presenting or phagocytic cells like dendritic cells and B cells. Future studies will examine this further.

With these results, we proceeded to assess the theranostic potential of nanoemulsion in vivo in an inflammation setting and validated its capacity to deliver therapeutics to macrophages and monitor the subsequent therapeutic response using fluorescence imaging. We evaluated this 
effect by comparing changes in fluorescence from inflamed paws between free drug and drug encapsulated in the nanoemulsion. In order to visualize macrophage accumulation changes in the group receiving free drug, a drug-free nanoemulsion, DFNE (vehicle), was also co-administered. Based on the live animal imaging at different time points, a visible difference in fluorescence intensity was seen between the groups with time (Fig. 7A). For both groups, we did not observe statistically significant differences in the ratio of fluorescence intensity between inflamed paw and control paw until $10 \mathrm{~h}$ (Fig. 7B). At the $24 \mathrm{~h}$ time point, a significant reduction in the fluorescence ratio was observed for the group receiving CXBNE, which was further reduced at $34 \mathrm{~h}$ time point, and persisted through $72 \mathrm{~h}$ (Fig. 7B). In the animals receiving free drug, paw fluorescence remained high at all the time points. ${ }^{19} \mathrm{~F}$ NMR of excised paws showed characteristic peaks corresponding to PFPE (Fig. S11), validating the presence of nanoemulsion at the inflammation site. The fluorescence intensity was assessed for both the nanoemulsions (Fig. S12) in order to exclude the contribution of differential nanoemulsion fluorescence to the observed fluorescence changes between the animal groups. Fluorescence intensity was observed to be slightly higher in CXBNE, which further supports the claim that animal group receiving CXBNE showed reduced fluorescence with time compared to the group receiving DFNE.

The biodistribution study revealed that the highest non-target organs that accumulated nanoemulsion were the liver and spleen (Fig. 8A). This result is expected because these organs are sites for a large macrophage presence. Interestingly, the percent injected dose per gram $(\% \mathrm{ID} / \mathrm{g})$ that accumulated in the spleen was higher for the group receiving CXBNE compared to the free drug (Fig. 8B). Recently, it has been reported that monocytes/macrophages from spleen, in addition to blood monocytes, are recruited to the injured site during inflammation [34]. We speculate that the increased nanoemulsion fluorescence in spleen for these animals is due to the 
reduced recruitment of nanoemulsion-internalized monocytes/macrophages to the inflamed paw. This result also coincides with the reduced macrophage infiltration of the inflamed paw in CXBNE group compared to the free drug group (Fig. 7B).

Histological analysis of the inflamed paw revealed a high level of macrophage infiltration (Fig. 9). We observed that the majority of the nanoemulsion droplets are co-localized with macrophages $(\mathrm{CD} 68+)$ expressing the target enzyme, COX-2. Further, CXBNE and DFNE showed accumulation specifically in macrophages compared to neutrophils (Fig. S13), similar to the histology data from $24 \mathrm{~h}$ after inflammation (Fig. 6). The combination of in vivo imaging and histological studies confirm that the nanoemulsion is efficiently taken up by macrophages in the inflamed tissue. In vivo live animal data indicate that by delivering celecoxib in a nanoemulsion, macrophage infiltration is reduced over time. We showed that the theranostic system not only delivered celecoxib to the target cells, but also facilitated the visualization of therapeutic response (reduced macrophage infiltration). Most importantly, the combined therapy and response monitoring was achieved after a single dose administration of the CXBNE.

\section{Conclusions}

We developed a stable, non-toxic, theranostic nanoemulsion that can be internalized by macrophages in vitro and in vivo. The nanoemulsions accumulated at the inflamed site specifically in macrophages, which can be detected by NIRF and ${ }^{19} \mathrm{~F}$ NMR imaging. The theranostic systems enabled us to monitor changes in macrophage infiltration in response to therapeutic delivery after a single dose administration. This multifunctional platform can be modified to incorporate other hydrophobic drugs to be applied in different disease models that 
possess an inflammatory component. Future work will focus on evaluating different dosing regimens for effective disease management.

\section{Acknowledgements}

Authors thank Dr. Lauren Ernst from Carnegie Mellon University for help with fluorescence measurements, Ms. Sneha Potdar from Duquesne University for assistance with protein assay, Mr. Lu Liu from Duquesne University for performing DLS measurements and Ms. Christina Bagia from Duquesne University for her assistance in solubility studies. This research was funded in part by Duquesne University Mylan School of Pharmacy startup funds (J.M.J.), grant from Commonwealth Universal Research Enhancement (CURE) program from the Pennsylvania Department of Health (JMJ and SKP), Pittsburgh Tissue Engineering Initiative Seed Grant (JMJ and John A. Pollock), the Bill and Melinda Gates Foundation TB Drug Accelerator Grant (\#OPP1034408 to JoAnne Flynn and CJA). University of Pittsburgh Cancer Institute (UPCI) shared resources (In Vivo Imaging Facility) were used in this research, and this facility is

supported in part by award NCI P30CA047904. ${ }^{19} \mathrm{~F}$ NMR performed at Center for Molecular Analysis (Carnegie Mellon University) supported by NSF (Grant numbers CHE-0130903 and CHE-1039870). Funding sources had no role in the study design, collection and interpretation of the data, preparation of manuscript or decision to submit the paper for publication.

\section{References}

[1] I. Tabas, C.K. Glass, Anti-inflammatory therapy in chronic disease: challenges and opportunities, Science, 339 (2013) 166-172.

[2] R. Noy, J.W. Pollard, Tumor-associated macrophages: from mechanisms to therapy, Immunity, 41 (2014) 49-61. 
[3] J.M. Olefsky, C.K. Glass, Macrophages, inflammation, and insulin resistance, Annual review of physiology, 72 (2010) 219-246.

[4] K.J. Moore, I. Tabas, Macrophages in the pathogenesis of atherosclerosis, Cell, 145 (2011) 341-355.

[5] Y. Ma, R.M. Pope, The role of macrophages in rheumatoid arthritis, Current pharmaceutical design, 11 (2005) 569-580.

[6] P.J. Murray, T.A. Wynn, Protective and pathogenic functions of macrophage subsets, Nature Reviews Immunology, 11 (2011) 723-737.

[7] B. Ajami, J.L. Bennett, C. Krieger, K.M. McNagny, F.M. Rossi, Infiltrating monocytes trigger EAE progression, but do not contribute to the resident microglia pool, Nature neuroscience, 14 (2011) 1142-1149.

[8] R. Weissleder, M. Nahrendorf, M.J. Pittet, Imaging macrophages with nanoparticles, Nature materials, 13 (2014) 125-138.

[9] B. Brochet, M. Deloire, T. Touil, O. Anne, J. Caille, V. Dousset, K. Petry, Early macrophage MRI of inflammatory lesions predicts lesion severity and disease development in relapsing EAE, Neuroimage, 32 (2006) 266-274.

[10] A. Balducci, B.M. Helfer, E.T. Ahrens, C.F. O’Hanlon, A.K. Wesa, Visualizing arthritic inflammation and therapeutic response by fluorine-19 magnetic resonance imaging (19F MRI), J. Inflammation (London, UK), 9 (2012) 24-34.

[11] S.K. Patel, J.M. Janjic, Macrophage Targeted Theranostics as Personalized Nanomedicine Strategies for Inflammatory Diseases, Theranostics, 5 150-172.

[12] J.R. McCarthy, E. Korngold, R. Weissleder, F.A. Jaffer, A light-activated theranostic nanoagent for targeted macrophage ablation in inflammatory atherosclerosis, Small, 6 (2010) 2041-2049.

[13] S. Cho, O. Hwang, I. Lee, G. Lee, D. Yoo, G. Khang, P.M. Kang, D. Lee, Chemiluminescent and Antioxidant Micelles as Theranostic Agents for Hydrogen Peroxide Associated-Inflammatory Diseases, Advanced Functional Materials, 22 (2012) 4038-4043.

[14] T. Harel-Adar, T.B. Mordechai, Y. Amsalem, M.S. Feinberg, J. Leor, S. Cohen, Modulation of cardiac macrophages by phosphatidylserine-presenting liposomes improves infarct repair, Proceedings of the National Academy of Sciences, 108 (2011) 1827-1832.

[15] E. Cittadino, M. Ferraretto, E. Torres, A. Maiocchi, B.J. Crielaard, T. Lammers, G. Storm, S. Aime, E. Terreno, MRI evaluation of the antitumor activity of paramagnetic liposomes loaded 
with prednisolone phosphate, European journal of pharmaceutical sciences : official journal of the European Federation for Pharmaceutical Sciences, 45 (2012) 436-441.

[16] D.K. Kadayakkara, S. Ranganathan, W.-B. Young, E.T. Ahrens, Assaying macrophage activity in a murine model of inflammatory bowel disease using fluorine-19 MRI, Laboratory Investigation, 92 (2012) 636-645.

[17] A. Balducci, Y. Wen, Y. Zhang, B.M. Helfer, T.K. Hitchens, W.S. Meng, A.K. Wesa, J.M. Janjic, A novel probe for the non-invasive detection of tumor-associated inflammation, Oncoimmunology, 2 (2013) e23034.

[18] K. Vasudeva, K. Andersen, B. Zeyzus-Johns, T.K. Hitchens, S.K. Patel, A. Balducci, J.M. Janjic, J.A. Pollock, Imaging Neuroinflammation In Vivo in a Neuropathic Pain Rat Model with Near-Infrared Fluorescence and 19F Magnetic Resonance, PloS one, 9 (2014) e90589.

[19] B. Ebner, P. Behm, C. Jacoby, S. Burghoff, B.A. French, J. Schrader, U. Flögel, Early assessment of pulmonary inflammation by 19F MRI in vivo, Circulation: Cardiovascular Imaging, 3 (2010) 202-210.

[20] P.K. Bae, J. Jung, S.J. Lim, D. Kim, S.-K. Kim, B.H. Chung, Bimodal perfluorocarbon nanoemulsions for nasopharyngeal carcinoma targeting, Molecular Imaging and Biology, 15 (2013) 401-410.

[21] S.K. Patel, Y. Zhang, J.A. Pollock, J.M. Janjic, Cyclooxgenase-2 Inhibiting Perfluoropoly (Ethylene Glycol) Ether Theranostic Nanoemulsions-In Vitro Study, PloS one, 8 (2013) e55802.

[22] L. Minghetti, Cyclooxygenase-2 (COX-2) in inflammatory and degenerative brain diseases, Journal of Neuropathology \& Experimental Neurology, 63 (2004) 901-910.

[23] G.A. FitzGerald, COX-2 and beyond: Approaches to prostaglandin inhibition in human disease, Nature reviews. Drug discovery, 2 (2003) 879-890.

[24] E. Hahn, S. Kraus, N. Arber, Role of cyclooxygenase-2 in pathogenesis and prevention of colorectal cancer, Digestive Diseases, 28 (2010) 585-589.

[25] P. Kalinski, Regulation of immune responses by prostaglandin E2, Journal of immunology, 188 (2012) 21-28.

[26] E. Ricciotti, G.A. FitzGerald, Prostaglandins and inflammation, Arteriosclerosis, thrombosis, and vascular biology, 31 (2011) 986-1000.

[27] I. Gomez, N. Foudi, D. Longrois, X. Norel, The role of prostaglandin E<sub $>2</$ sub $>$ in human vascular inflammation, Prostaglandins, Leukotrienes and Essential Fatty Acids (PLEFA), 89 (2013) 55-63. 
[28] J.G. Barin, G.C. Baldeviano, M.V. Talor, L. Wu, S. Ong, F. Quader, P. Chen, D. Zheng, P. Caturegli, N.R. Rose, D. Cihakova, Macrophages participate in IL-17-mediated inflammation, European journal of immunology, 42 (2012) 726-736.

[29] S.K. Patel, M.J. Patrick, J.A. Pollock, J.M. Janjic, Two-color fluorescent (near-infrared and visible) triphasic perfluorocarbon nanoemulsions, Journal of biomedical optics, 18 (2013)

101312-101312.

[30] K. Konopka, N. Overlid, A.C. Nagaraj, N. Duzgunes, Serum decreases the size of Metafectene-and Genejammer-DNA complexes but does not affect significantly their transfection activity in SCCVII murine squamous cell carcinoma cells, Cellular \& molecular biology letters, 11 (2006) 171-190.

[31] G.E. Gerhardt, R.J. Lagow, Synthesis of the perfluoropoly (ethylene glycol) ethers by direct fluorination, The Journal of Organic Chemistry, 43 (1978) 4505-4509.

[32] T. Wang, J. Bai, X. Jiang, G.U. Nienhaus, Cellular uptake of nanoparticles by membrane penetration: a study combining confocal microscopy with FTIR spectroelectrochemistry, ACS nano, 6 (2012) 1251-1259.

[33] I. Raynal, P. Prigent, S. Peyramaure, A. Najid, C. Rebuzzi, C. Corot, Macrophage endocytosis of superparamagnetic iron oxide nanoparticles: mechanisms and comparison of ferumoxides and ferumoxtran-10, Investigative radiology, 39 (2004) 56-63.

[34] F.K. Swirski, M. Nahrendorf, M. Etzrodt, M. Wildgruber, V. Cortez-Retamozo, P. Panizzi, J.L. Figueiredo, R.H. Kohler, A. Chudnovskiy, P. Waterman, E. Aikawa, T.R. Mempel, P. Libby, R. Weissleder, M.J. Pittet, Identification of splenic reservoir monocytes and their deployment to inflammatory sites, Science, 325 (2009) 612-616. 


\section{Figure Legends}

Fig. 1. (A) Size distribution of nanoemulsions NE1 (red), NE2 (blue), and NE3 (green). (B) Nanoemulsion droplet diameter changes with time stored at $60{ }^{\circ} \mathrm{C}$. Storage stability of nanoemulsions (C) NE1, (D) NE2, and (E) NE3 stored at 4, 25 and $37^{\circ} \mathrm{C}$. Standard deviations represent half width of polydispersity index (PDIw/2).

Fig. 2. Characterization of the optimized fluorescent dye-loaded nanoemulsions, DFNE and CXBNE. (A) Zeta potential distribution of DFNE (red) and CXBNE (green). (B) Graph showing droplet diameter changes with time (days) for nanoemulsions stored at $4{ }^{\circ} \mathrm{C},(\mathrm{C})$ with serum containing cell culture media at $37^{\circ} \mathrm{C}$, and (D) pH 5.0 and 7.4 at $37^{\circ} \mathrm{C}$. (E) Fluorescence excitation and (F) Emission spectra.

Fig. 3. (A) Viability of RAW 264.7 macrophages exposed to DFNE and CXBNE for $24 \mathrm{~h}$. Data is shown as mean of percent unexposed cells $\pm S D(n=4)$. (B) Nitric oxide release from macrophages exposed to nanoemulsions for $24 \mathrm{~h}$. (C) CD86 expression in macrophages exposed to nanoemulsions for $24 \mathrm{~h}$. Cells not exposed to any treatment are designated as NT (no treatment).

Fig. 4. Nanoemulsion uptake by macrophages. (A) Comparison of fluorescence emission of cells treated with or without nanoemulsion showing emission maximum at $670 \mathrm{~nm}$. (B) Confocal microscopy of cells incubated with nanoemulsion $(10 \mu \mathrm{L} / \mathrm{mL})$ for $24 \mathrm{~h}$ at $37^{\circ} \mathrm{C}$. Merged differential interference contrast (DIC) image shows cell morphology with incorporated fluorescent nanoemulsions. (C) Confocal images showing co-localization of nanoemulsion (red) 
with acidic compartments (green) labeled with Lysotracker Green-DND. Merged image show the co-localized pixels (yellow). Nuclei are stained with DAPI (blue).

Fig. 5. (A) Confocal images of cells exposed to nanoemulsion for 5, 15, 30, and $60 \mathrm{~min}$ incubated at 4 and $37{ }^{\circ} \mathrm{C}$. (B) Dose dependent uptake of nanoemulsion in macrophages. Dose is represented on $\mathrm{x}$-axis as PFPE $(\mathrm{mg} / \mathrm{mL})$. (C) Time-dependent uptake of nanoemulsion $(4 \mathrm{mg} / \mathrm{ml}$ PFPE) in macrophages. (D) Comparison of uptake between cells exposed to different doses of nanoemulsion with or without phagocytosis inhibitor Cytochalasin B $(5 \mathrm{ug} / \mathrm{mL})$. Uptake was performed for $2.5 \mathrm{~h}$. Data in panels B, C, and D is from triplicates expressed as mean $\pm \mathrm{SD}$.

Fig. 6. Representative immunofluorescence sections of inflamed paw and spleen from mouse injected with nanoemulsion $24 \mathrm{~h}$ post-inflammation. Nanoemulsion was injected $12 \mathrm{~h}$ preinflammation. Sections were stained with anti-CD68 for macrophages and anti-Gr-1 for neutrophils. Merge images shows colocalization (arrows) of the nanoemulsion (red) with macrophages (green) in inflamed paw and spleen and no colocalization (arrow head) of nanoemulsion (red) with neutrophils (green).

Fig. 7. (A) Representative fluorescence/X-ray images showing the accumulation of nanoemulsion in the site of inflammation (right paw) at different time points. Inflammation was induced by injection of CFA $(50 \mu \mathrm{L})$ subcutaneous at the right paw. Treatments were injected 12 $\mathrm{h}$ prior to inducing inflammation. (B) Ratio of quantified fluorescence of inflamed paw compared to non-inflamed paw $(\mathrm{n}=5 ;(* * *) \mathrm{p}<0.001)$.

Fig. 8. Biodistribution of theranostic nanoemulsion. (A) Representative fluorescence images of different organs showing nanoemulsion biodistribution at $72 \mathrm{~h}$ after inducing inflammation 
evaluated by fluorescence ex vivo imaging. (B) Biodistribution of nanoemulsion in different organs $(\mathrm{n}=3 ;(* *) \mathrm{p}<0.01)$.

Fig. 9. Representative immunofluorescence sections of inflamed paw from mouse injected with DFNE and CXBNE (purple) and stained for macrophages (Rat anti mouse-CD68, green) and COX-2 (Goat anti mouse-COX-2, red). Merged panel shows the colocalization of nanoemulsion fluorescence (DiD) with macrophages and COX-2 staining. 
Uptake by

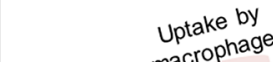

no drug Blood vessel

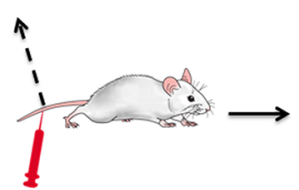

COX-2 inhibiting theranostic nanoemulsion

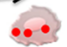

$\longrightarrow$

CFA injection

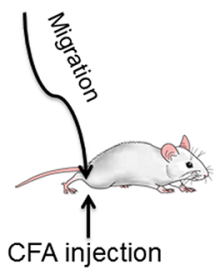

Graphical Abstract
Imaging macrophage infiltration

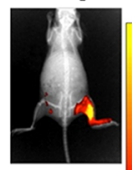

$8 \times 10^{8}$
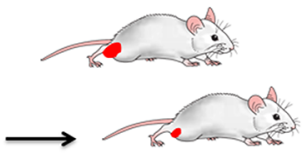

w drug

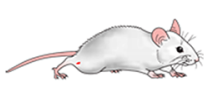

Inflammation response

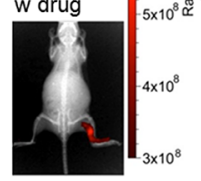


A Figure 1

Size Distribution by Intensity

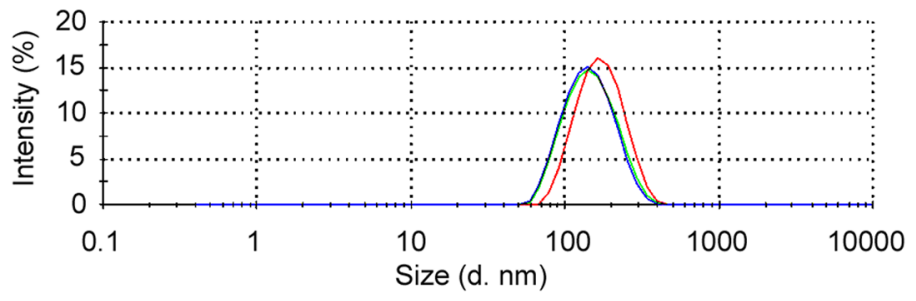

\section{C}

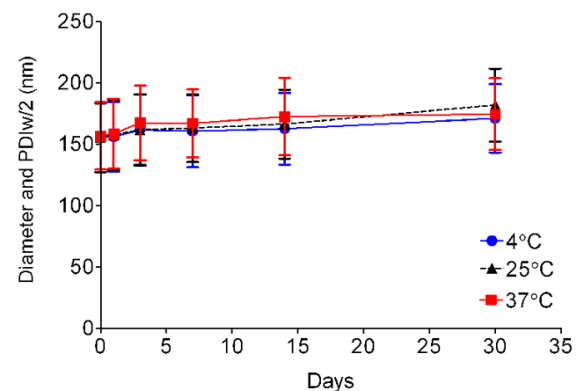

D

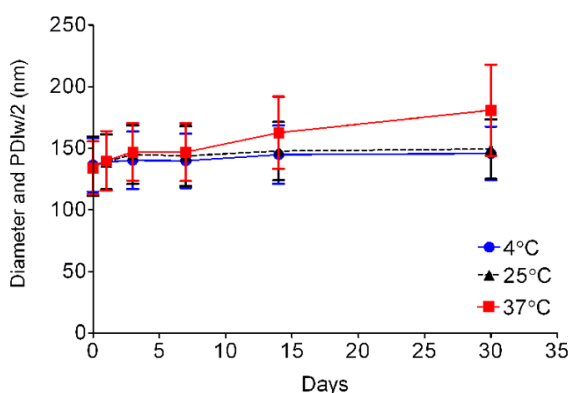

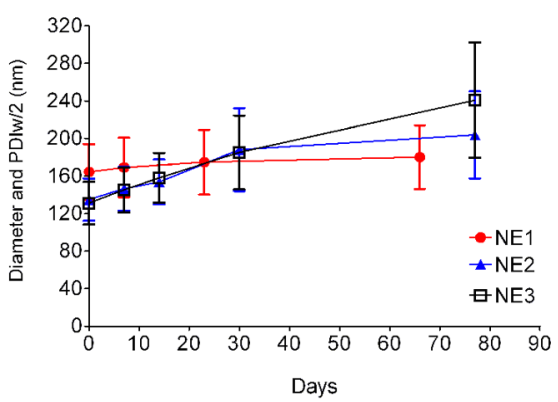

E

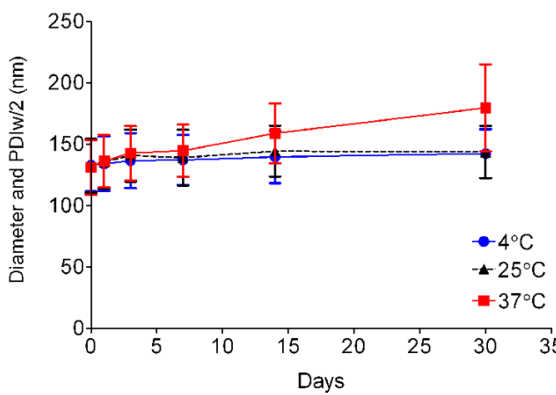


A $\quad$ Figure 2

B
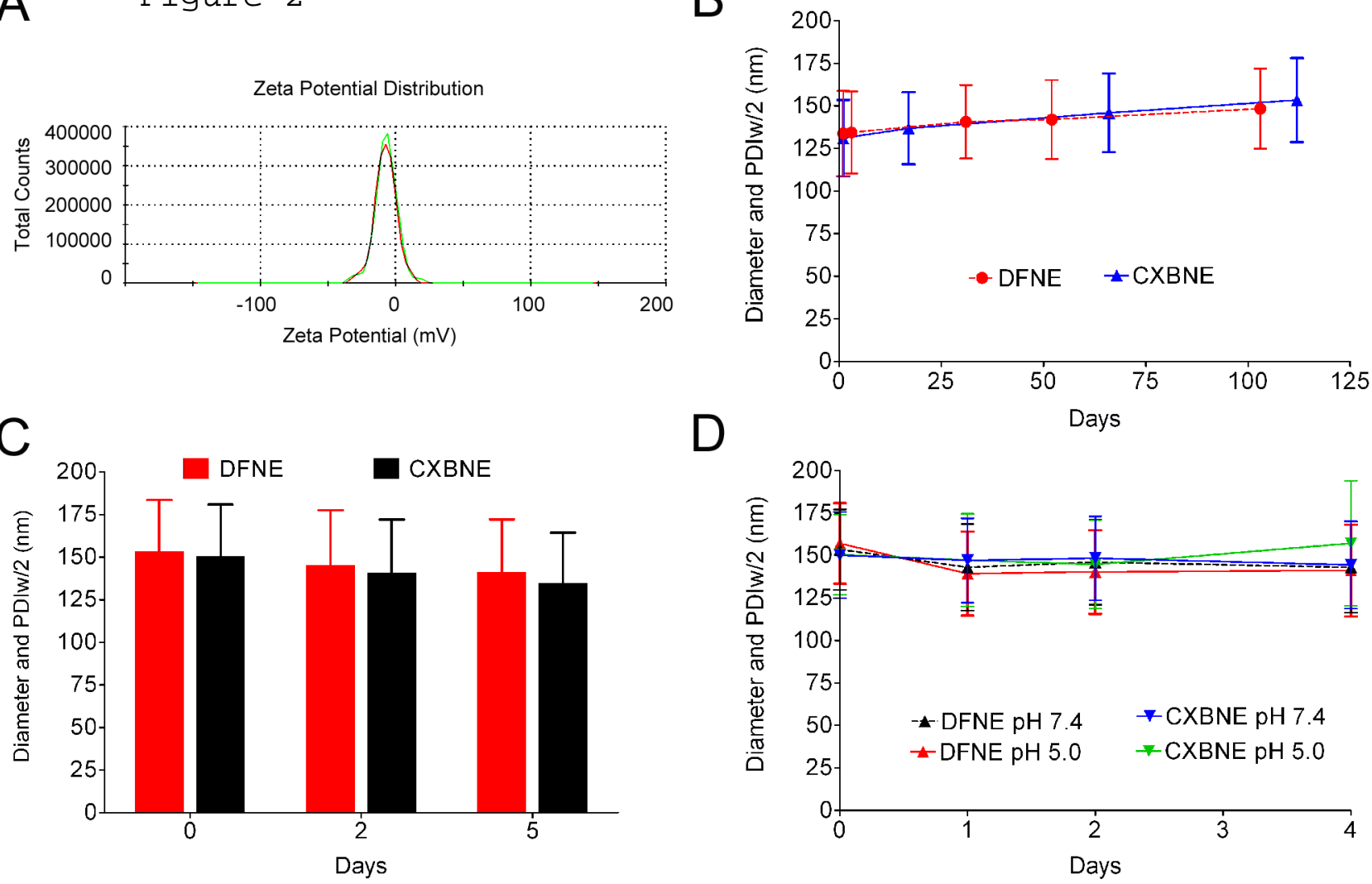

E

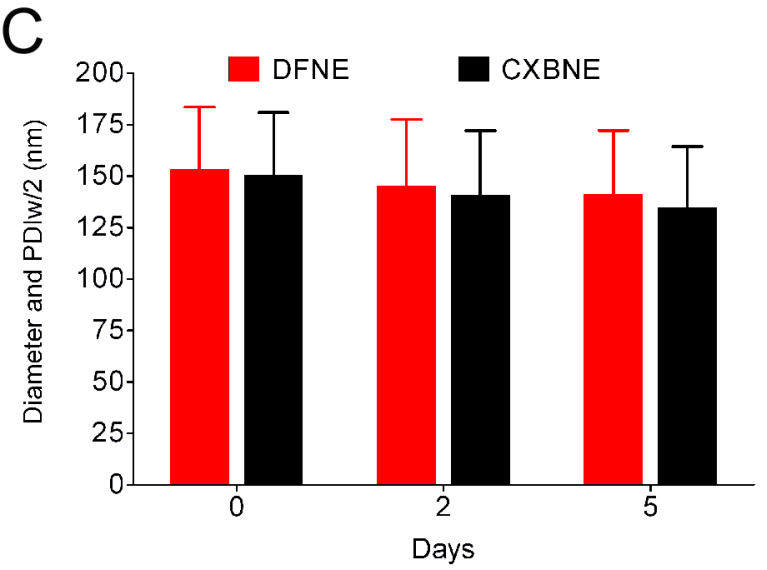

F
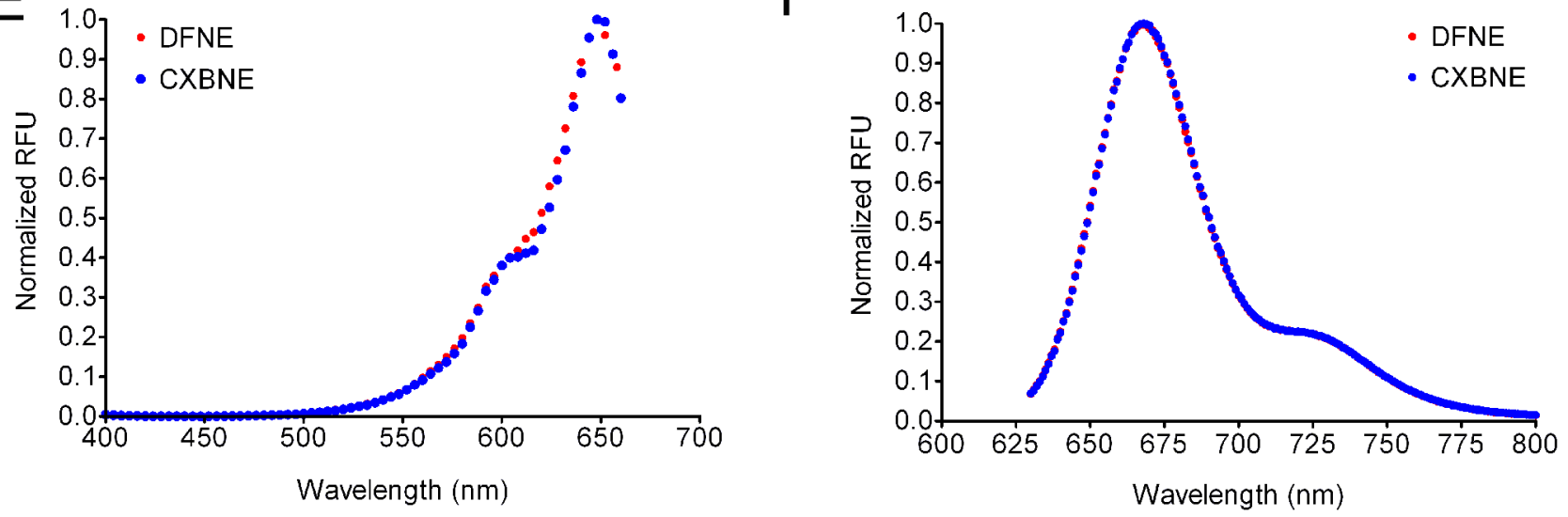


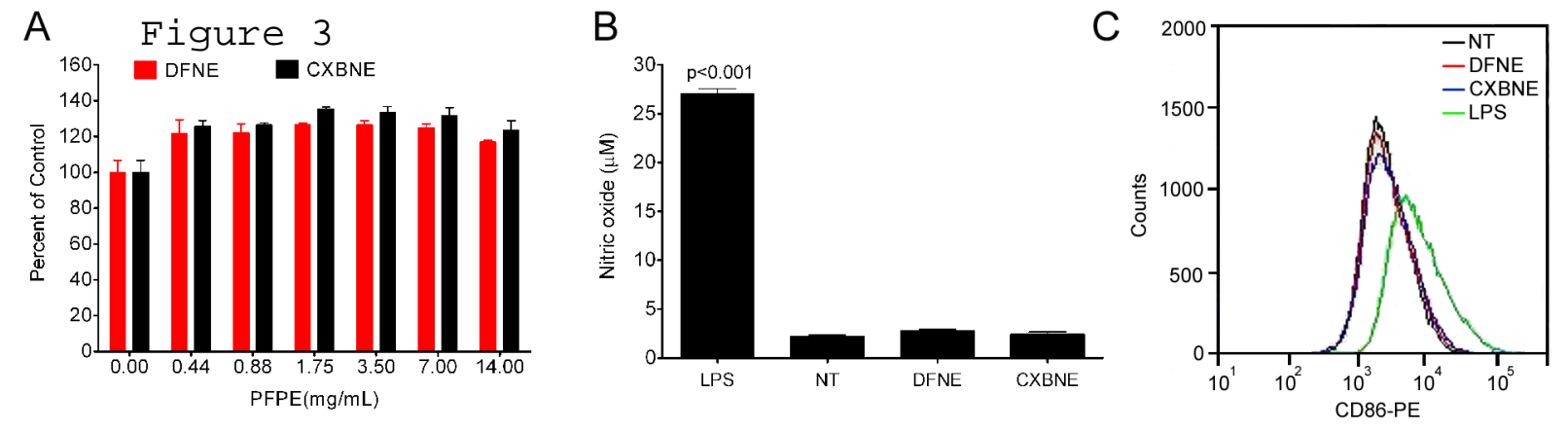


A $\quad$ Figure 4

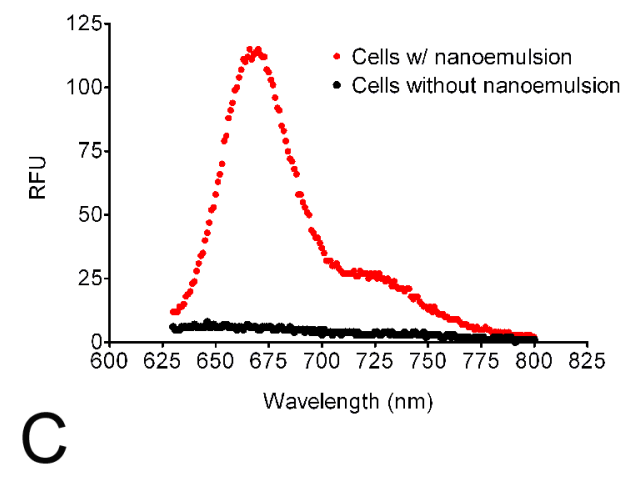

Lysotracker Green DND-26
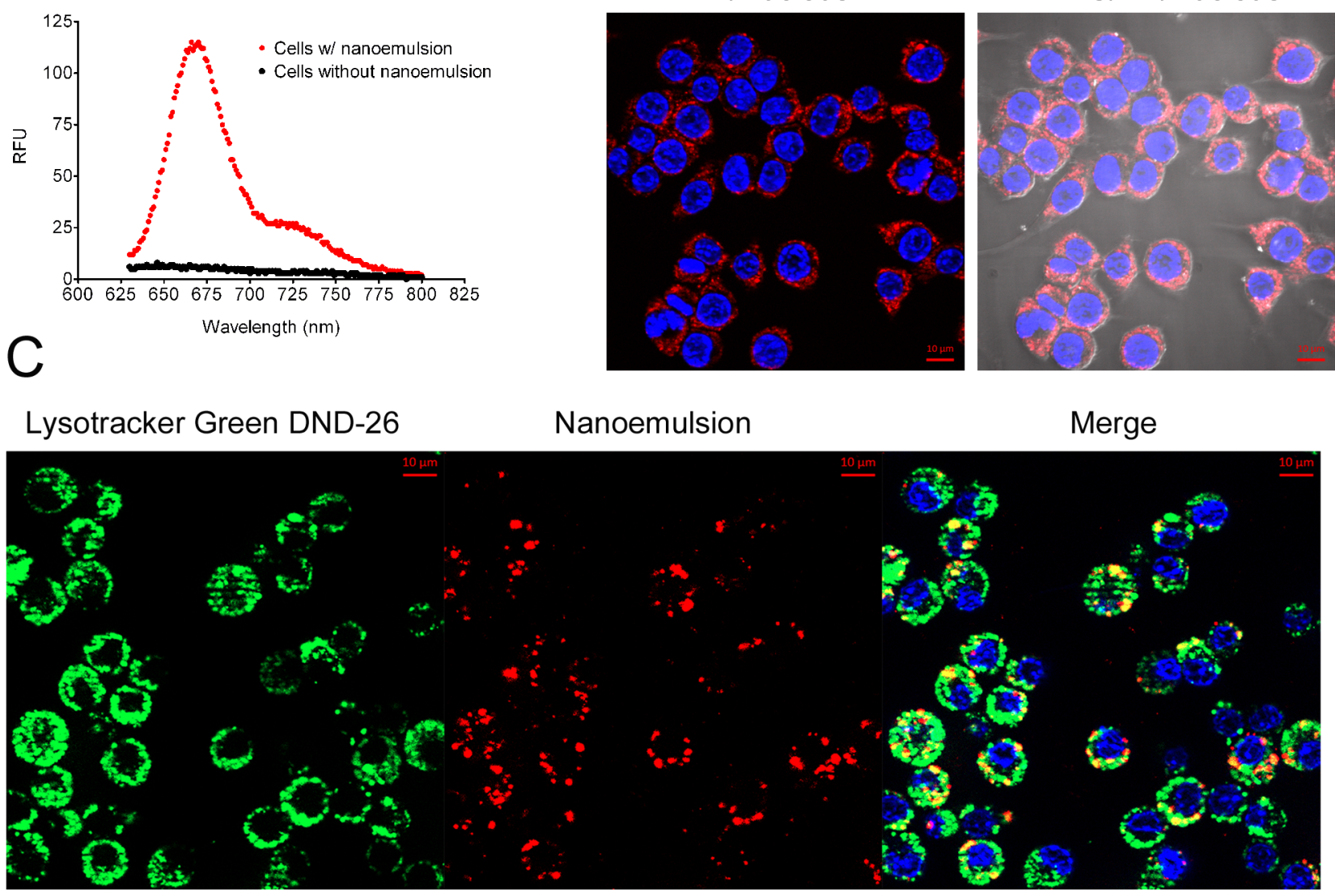

NE/Nucleus

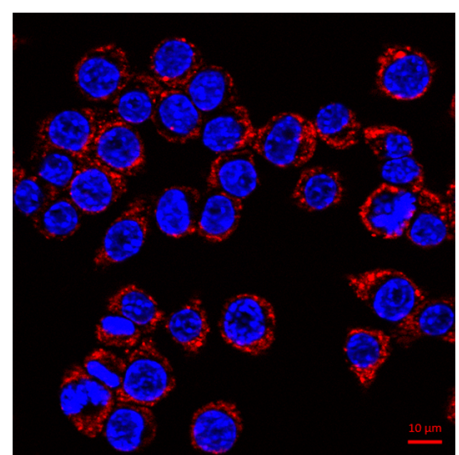

Nanoemulsion

Merge
DIC/NE/Nucleus
B 

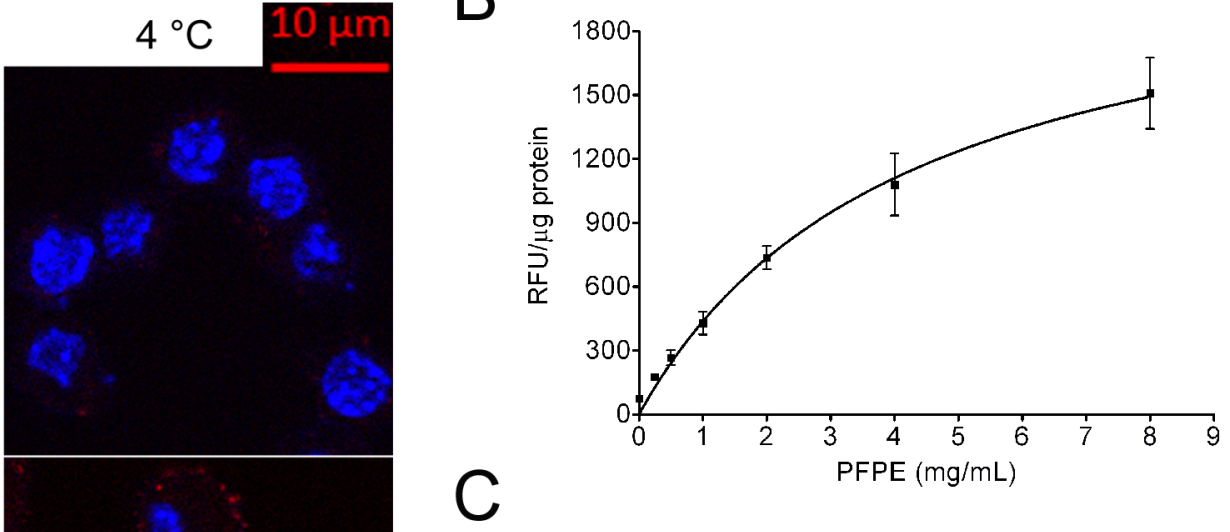

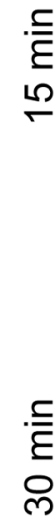

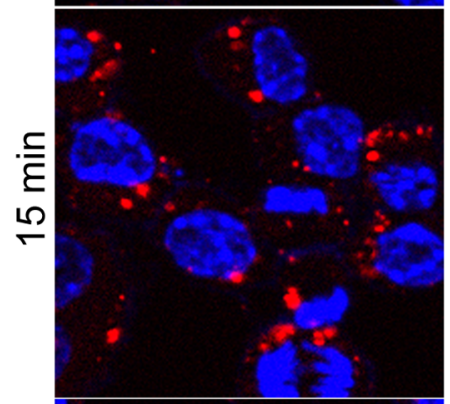

C

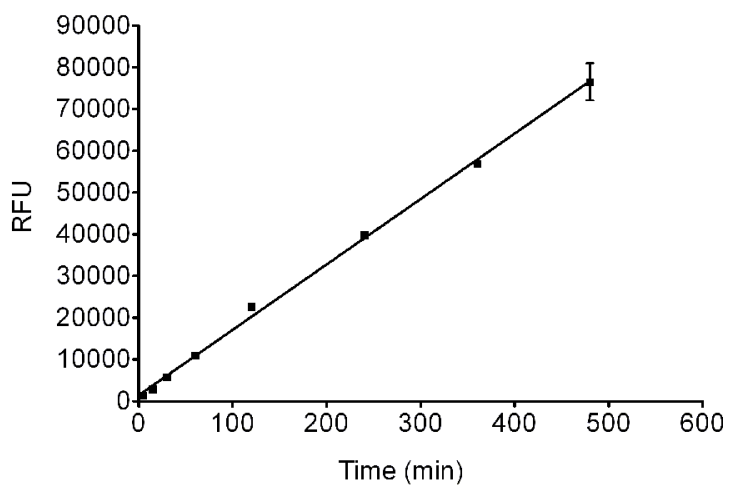

D

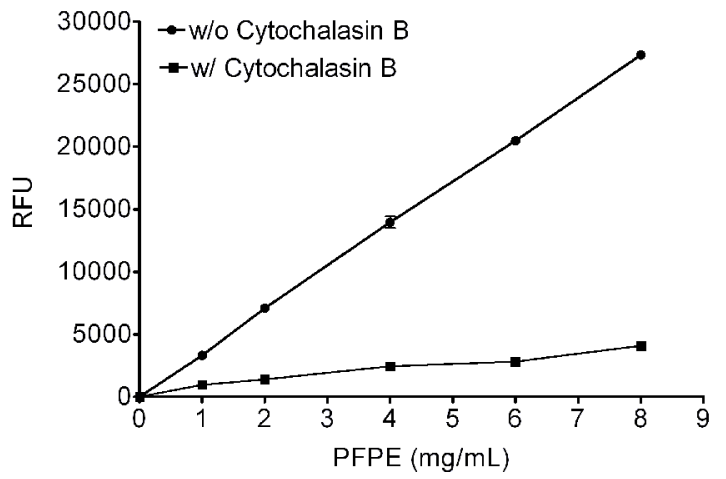

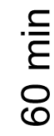
PFPE $(\mathrm{mg} / \mathrm{mL})$ 

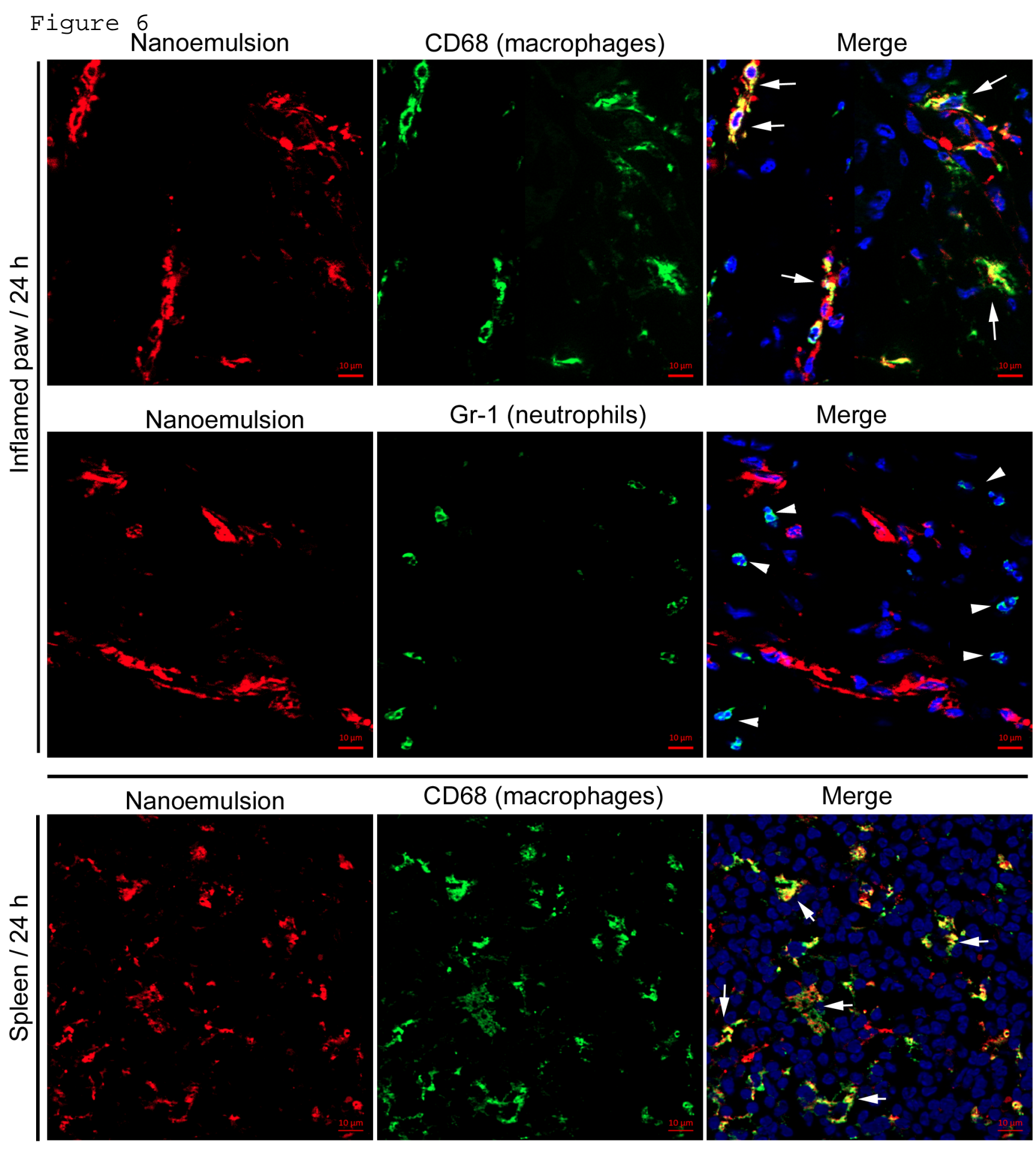


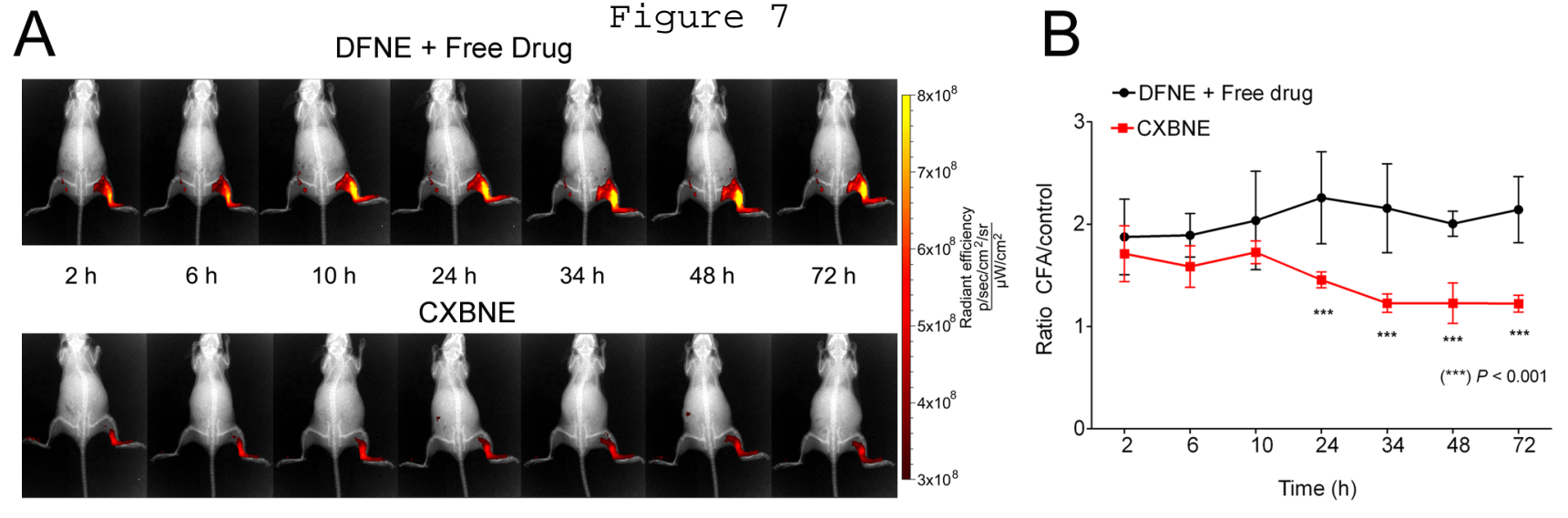




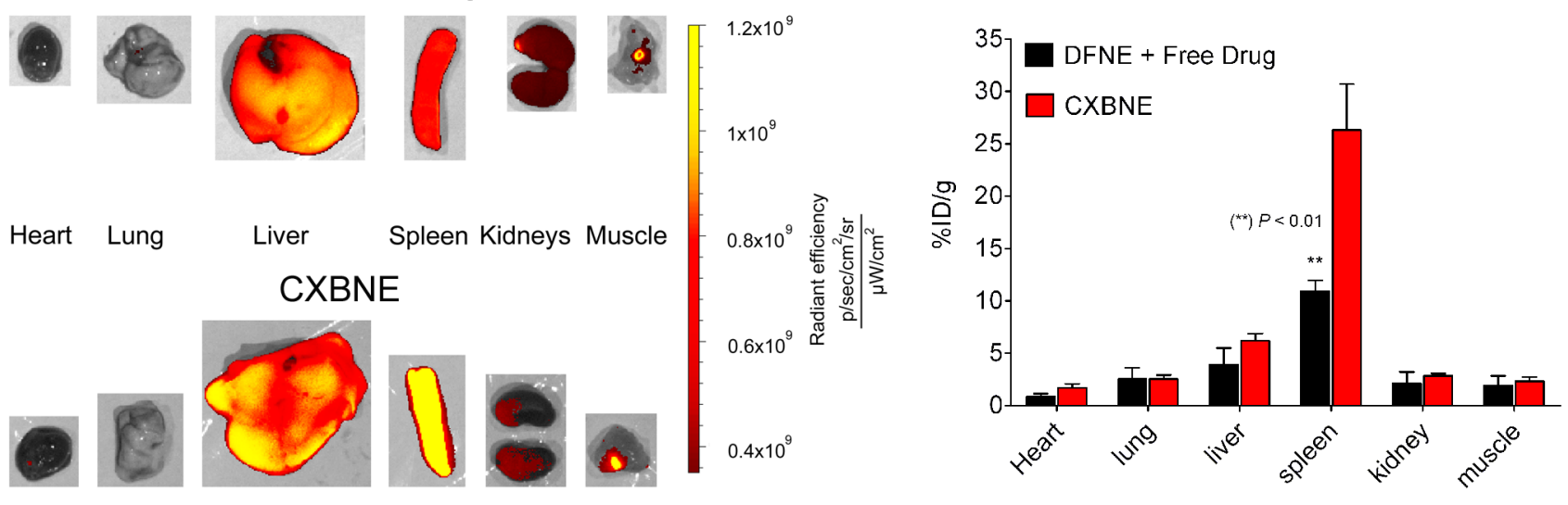




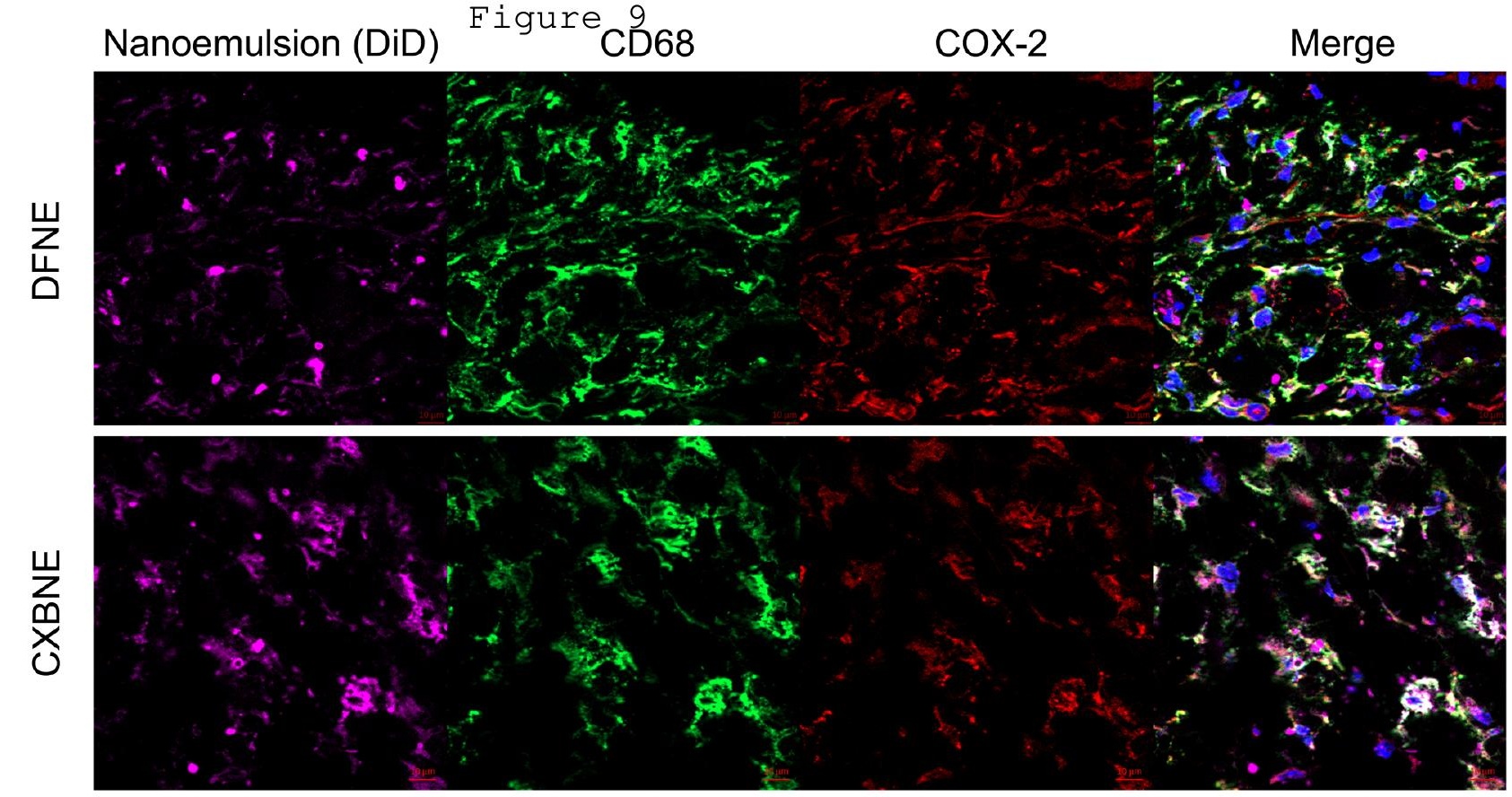


Table 1

DLS characterization of nanoemulsions NE1, NE2 and NE3

\begin{tabular}{cccccccc}
\hline Code & $\begin{array}{c}\text { PFPE } \\
(\% \text { w/v) }\end{array}$ & $\begin{array}{c}\text { Surfactant } \\
(\% \text { w/v) }\end{array}$ & Pulses & O/S $^{\text {a }}$ & $\begin{array}{c}\text { Size } \\
(\mathbf{n m})\end{array}$ & PDI & $\begin{array}{c}\text { Zeta potential } \\
(\mathbf{m V})\end{array}$ \\
\hline NE1 & 18.75 & 3.75 & 38 & 5.96 & 156 & 0.12 & -14.0 \\
NE2 & 18.75 & 2 & 30 & 11.17 & 136 & 0.10 & -12.1 \\
NE3 & 18.75 & 2 & 46 & 11.17 & 133 & 0.10 & -12.8 \\
\hline *O/S = Oil to surfactant ratio. Oil phase includes Miglyol 810N and PFPE oxide
\end{tabular}

